\title{
Induction of Univalent Chromosomes in Explanted Lily Microsporocytes by Inhibitors of DNA Synthesis
}

\author{
Miyako H. Takegami \\ Department of Biology, Faculty of Science, Nagoya University, Chikusa-ku, \\ Nagoya 464, Japan
}

\begin{abstract}
The cytological effects of nalidixic acid and novobiocin, inhibitors of DNA synthesis, on meiotic division in explanted microsporocytes of Lilium longiflorum were examined. Microsporocytes in the late $\mathrm{G}_{2}$ phase of premeiosis and in the early meiotic prophase were cultured in vitro for discrete periods in the presence of various concentrations of one of these inhibitors. The main effects of $0.4 \mathrm{mM}$ nalidixic acid applied during leptonema and zygonema were suppression of meiotic development and the production of sticky chromosomes. At 0.1 to $0.2 \mathrm{mM}$, the meiotic rate was greatly reduced, and an application to cells in late $\mathrm{G}_{2}$ phase or early leptonema greatly affected chromosome pairing and chiasma formation, eventually giving rise to univalent or highly achiasmatic chromosomes.

Electron microscopy showed that nalidixic acid does not interfere with ongoing synapsis, but has its effect on chiasma formation by suppressing the initiation of formation of synaptinemal complexes. These results are evidence that this drug interferes with the mechanism of initiation of synapsis, but not with the maintenance of chromosome integrity. Similar results were obtained with microsporocytes exposed to novobiocin at concentrations of 0.005 to $0.02 \mathrm{mM}$. The significance of DNA synthesis during the zygotene stage is discussed on the basis of these observations.
\end{abstract}

In several organisms, particularly in Lilium and Trillium, an integral part of the nuclear DNA fails to replicate during the $\mathrm{S}$ phase of premeiosis, its replication being delayed until zygonema when chromosomes undergo pairing $(4,5,6,15)$. The synthesis of this DNA component, referred to as zDNA, is considered essential for normal meiotic development (15). The main effects found when DNA synthesis is inhibited during zygonema are marked suppression of meiotic development and the product on of chromosomal aberrations $(7,13,14,18)$.

Electron microscopy studies have shown that zDNA synthesis takes place close to, or within, the synaptinemal complex (SC), a unique structure associated with chromatin during synapsis (10) and that the formation of this structure is prevented in cells arrested by DNA-inhibitory agents (12). These reports have adequately shown that $\mathrm{zDNA}$ is a factor in the pairing process and that some DNA synthesis is involved in formation of the SC.

Abbreviations used: AdR, deoxyadenosine; HU, hydroxyurea; NAL, nalidixic acid; NOV, novobiocin; SC, synaptinemal complex; zDNA, zygotene DNA. 
However, questions remain as to what role zDNA plays in the synaptic event. Information on the functional significance of this synthesis in relation to meiotic development should be produced from detailed cytological analyses of the meiotic process in the presence of various inhibitors of DNA synthesis. For this reason we applied inhibitors to explanted lily mircosporocytes in different stages of meiosis and examined the cytological consequences.

Certain types of abnormalities in chromosomes and meiotic development were produced in the meiocytes; these were related to the stage at which the inhibitors were administered (13). The types of abnormalities in leptonema cells were divisible into two broad groups: total arrest of meiotic development with inhibition of SC formation and the suppression of development beyond prophase but with sustained zygonema development to formation of the SC structure. The former is characteristic of cells exposed to several inhibitors, one being deoxyadenosine (AdR) $(7,13)$. The latter effect is observed in cells exposed to an agent such as hydroxyurea (HU) (18). Both types of inhibitor produce strong inhibition of zDNA synthesis $(5,7)$, their apparent differences in relation to pairing being shown by the effects of these inhibitors.

Because of the effects of AdR and HU, it has been assumed that perturbation of scheduled DNA synthesis might affect the function of zDNA synthesis in the formation of the SC and eventually produce chromosome univalency. This assumption is reasonable because of the reversion to mitotic (asynaptic) division by preleptotene microsporocytes extruded into the culture media, in them premature replication of zDNA took place in the presynaptic phase $(9,19)$.

Applications of various inhibitors to meiocytes in the leptotene stage have produced two suitable drugs, nalidixic acid (NAL) and novobiocin (NOV), known specific inhibitors of DNA gyrase activity in prokaryotes (1). When applied to leptonema cells, these drugs effectively induced an asynaptic or highly achiasmatic condition. Whether the cells have similar susceptibility to the action of these drugs is not clear because we lack evidence on gyrase activity in the nuclei of higher plant cells. The development of successful methods for inducing univalent chromosomes in lily meiocytes treated with DNA-inhibitory agents, however, has shed some light on certain aspects of the synaptic process, as described here.

\section{MATERIALS AND METHODS}

Microsporocytes in various stages of meiosis were obtained from anthers of the lily, Lilium longiflorum var. Georgia. The procedure used to identify individual stages is based on the correlation between bud length and the meiotic stage $(2,19)$. A total of 24 cohesive strings of microsporocytes can be extruded from a bud. At the start of the experiment one or two strings from each bud were used for a chromosome squash preparation to determine the exact stage of meiosis. Sixteen strings from individual buds were cultured separately in the presence of NAL or NOV for up to 20 days. As controls, 6 or 7 strings were grown in culture medium without inhibitors.

At different times during culture, samples were taken. These cells were squashed in propionic orcein then scored cytologically for the stages and types of abnormalities. The methods used to culture microsporocytes have been described elsewhere $(8,19)$. Procedures used for electron microscopy were outlined in a previous paper (18).

The two inhibitors, NAL and NOV, were purchased from Sigma Chemical Company, and tested at concentrations of from 0.05 to $0.4 \mathrm{mM}$ for NAL and of from 0.005 to $0.02 \mathrm{mM}$ 
for NOV. The quantitative data given are from experiments with NAL unless otherwise stated.

\section{RESULTS}

General aspects of NAL action. The physiological response of meiotic cells to NAL largely corresponds to responses to other DNA-inhibitory agents $(13,18)$ and supports biochemical evidence that DNA is synthesized during meiotic prophase $(4,5)$. Inhibition of DNA synthesis causes such alterations in the general morphology of chromosomes as stickiness and fragmentation.

Characteristics of the effects of the inhibition of DNA synthesis during mieotic prophase that pertain directly to the effects of NAL found in this study are summarized thus: (1) The response of cells in leptonema and zygonema that are exposed to high concentrations of DNA-inhibitory agents is marked suppression of meiotic development. (2) When the inhibitors were used at half the minimum concentration for each agent that induced suppression, cells responded with a delay in meiotic progression, and could continue through the meiotic cycle. (3) The response of cells exposed to the inhibitors after late zygonema or early pachynema is the production of chromosomal aberrations. (4) When cells progress through pachynema in the presence of these drugs, damage suffered by their chromosomes is shown by stickiness.

When NAL was tested for its cytological effects on microsporocytes during meiotic prophase, it was essentially the HU-type with the characteristics given above. In the presence of this drug, the rate of meiotic development was reduced markedly and cytological abnormalities appeared. The type of abnormality produced depended on the stage of treatment, but the severity of the effect was a function of concentration. The relationship between the meiotic rate and the concentration of NAL in meiotic cells treated at early leptonema and early zygonema is shown in Fig. 1.

Because cells progress from the diplotene to tetrad stage at the rate of untreated
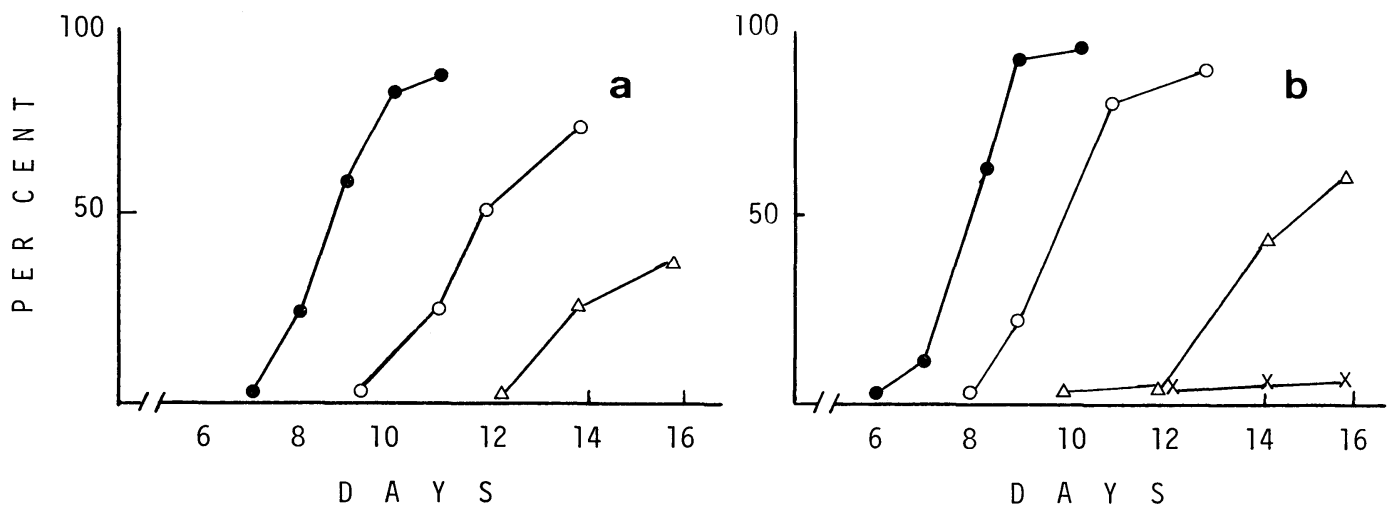

Fig. 1. Rate of meiotic development in the presence of various concentrations of NAL in microsporocytes of Lilium longiflorum explanted at late $\mathrm{G}_{2}$ to early leptonema (a) and at late leptonema to early zygonema (b). Meiotic development is expressed cumulatively as the percent of cells that have passed through metaphase $I$ in comparison to the total living cells. $\bullet$, control; $\bigcirc, 0.1 \mathrm{mM} \triangle \triangle, 0.2$ $\mathrm{mM} ; \times, 0.4 \mathrm{mM}$. 
cells, the rate of meiosis may be measured by cumulatively scoring cells that have gone through metaphase I. At a drug concentration of $0.1 \mathrm{mM}$, early leptonema cells required about 3 days longer to reach metaphase than the controls, and at $0.2 \mathrm{mM}$ they took 5-7 days longer to reach metaphase. In other experiments, the developmental period was useful for getting samples of NAL-treated meiocytes at the stage desired.

At the highest concentration used $(0.4 \mathrm{mM})$, meiotic development was markedly suppressed, even with further culture cells did not develop beyond prophase. These cells remained suspended in some stage of meiotic prophase, and died in culture after a period of about 20 days. Similar results were obtained with early zygonema cells (Fig. 1b).

The rate of meiosis was unaffected in cells exposed to NAL later than the midpachynema stage. These results indicate that cells undergoing DNA synthesis are slowed in their progress through prophase. Thus, regardless of the specific action of NAL, this drug interferes with the meiotic cycle when administered to cell undergoing DNA synthesis.

As stated above, the cytological abnormalities induced by NAL are both concentration and stage dependent. Responses of cells in various stages of meiosis to inhibitory concentrations of NAL are given in Table 1. Stage dependence is apparent from the response of the cells to the highest concentration of NAL, at which $(0.2 \mathrm{mM})$ development still takes place at an appreciable rate. One noticeable response of cells exposed to $0.2 \mathrm{mM}$ NAL earlier than late leptonema is that although they do reach metaphase their chromosomes are highly achiasmatic.

Similar phenomena were obtained with late $\mathrm{G}_{2}$ and early leptonema cells exposed to $0.1 \mathrm{mM}$ NAL. The production of the univalent chromosomes differs markedly from the response of cells treated with the DNA-inhibitory agent, HU or AdR. Inhibition of DNA synthesis by these drugs does not produce the chromosome univalency, induced by inhibitors of RNA and protein syntheses $(11,14)$. Thus, inhibition of DNA synthesis by NAL causes a unique alteration in chromosome pairing that produces

TABLE 1. CytologicAl EFFECTS OF NALIDIXIC ACID ON MEIOTIC CELLS

\begin{tabular}{|c|c|c|c|c|}
\hline \multirow{2}{*}{ Initial stage } & \multicolumn{4}{|c|}{ Concentration of nalidixic acid (mM) } \\
\hline & 0.05 & 0.1 & 0.2 & 0.4 \\
\hline Late $G_{2}$ to early leptonema & $\begin{array}{l}\text { partially } \\
\text { achiasmata }\end{array}$ & achiasmata & achiasmata & arrest \\
\hline Mid to late leptonema & chiasmata & chiasmata & $\underset{\text { (sticky) }}{\operatorname{achiasmata}}$ & $\begin{array}{c}\text { suspended } \\
\text { (sticky) }\end{array}$ \\
\hline Zygonema & chiasmate & chiasmata & $\begin{array}{c}\text { highly } \\
\text { chaismata } \\
\text { (sticky) }\end{array}$ & $\begin{array}{c}\text { suspended } \\
\text { (sticky) }\end{array}$ \\
\hline Early pachynema & - & - & chiasmata & $\begin{array}{c}\text { chiasmata } \\
\text { (sticky) }\end{array}$ \\
\hline Late pachynema & - & - & chiasmata & chiasmata \\
\hline
\end{tabular}

Cells were explanted at the stages indicated to culture medium containing 0.05 to $0.4 \mathrm{mM}$ nalidixic acid and cultured for up to 14 days. The types of effects show the abnormality typical for that initial stage of cells. Arrest: failed to develop beyond early prophase. Suspended: did not progress beyond, but stayed in, the late stage of prophase. Sticky: stickiness or fusion of bivalent and/or univalent chromosomes. - : not examined. 
an observable achiasmate condition.

Sticky chromosomes, the response of meiotic cells to several inhibitors of DNA synthesis, also appeared in cells treated with NAL. Cells in late leptonema to early pachynema that were exposed to $0.2 \mathrm{mM}$ NAL showed the lesion after 10 days of culture. Most cells progressed through metaphase I and later stages, the abnormality becoming observable in the clumping of chromosomes during metaphase or in the anaphase bridge.

Cells in leptonema and zygonema that were exposed to $0.4 \mathrm{mM}$, as stated above, remained suspended in meiotic prophase. When cultures were prolonged, these cells became necrotic and usually were accompanied by the clumping of their sticky chromosomes. This abnormality probably is not an immediate effect of NAL; but, as stated elsewhere $(7,19)$, we have no evidence of the primary lesion which leads to stickiness.

Effectiveness of NAL in causing chromosomal univalency. The appearance of univalent chromosomes is a unique response of cells exposed to NAL, in contrast to their responses to other DNA-inhibitory agents. This univalency is caused by the failure of the chiasma formation associated with two important events in meiosis, chromosome pairing and crossing over. I therefore studied the effectiveness of NAL on the induction of univalency.

When cells in the late $G_{2}$ phase of premeiosis were explanted under the given culture conditions, the chiasmate condition was highly stable, the mean frequency of cells with univalent chromosomes at metaphase I being within $20 \%$ of the total. Once cells entered leptonema they could be explanted without inhibiting chiasma formation; abnormal frequencies in these cells were less than $10 \%$ (9). But, cells exposed to $0.1 \mathrm{mM}$ NAL beginning in late $\mathrm{G}_{2}$ phase failed to form chiasmata (Table 1). Numerous comparisons were made of controls and NAL-treated meiocytes with respect to the chiasmate condition; all supported the effectiveness of NAL in causing univalency.

The relative frequency of cells with univalent chromosomes among the different groups of cell populations is given in Fig. 2. Cells with a mixture of univalents and bivalents also were scored, but the frequencies of these partially chiasmatic cells were relatively small. The frequency of achiasmatic cells decreased with the length of the leptotene stage, but this abnormality also was concentration dependent. At $0.2 \mathrm{mM}$, late leptonema cells failed to form chiasmata.

No achiasmatic response, however, was found in cells that had been exposed to NAL from early zygonema on. There is thus a critical stage in chiasmatic development beyond which inhibition of DNA synthesis by NAL does not effect chiasma formation, but does effect chromosome association and segregation in a variety of ways. A small degree of asynchrony in groups of microsporocytes obtained from the buds made it difficult to decide the exact explanted stages of the cultured cells. Repeated experiments, however, indicated the probability that only in cells in presynaptic stages can univalency be induced by NAL; ongoing pairing is unaffected.

The effects of NAL treatment on lily meiocytes are shown in Fig. 3. Unlike the controls, treated nuclei have significant amount of unpaired chromatin threads (Fig. 3a). Asynapsis often extends to the whole chromosome rather than only to segments, whereas pairing appears to be random.

Partial pairing would be effective for side-by-side association of univalents, but might not be effective for the formation of chiasmata. Such ineffective pairing that 

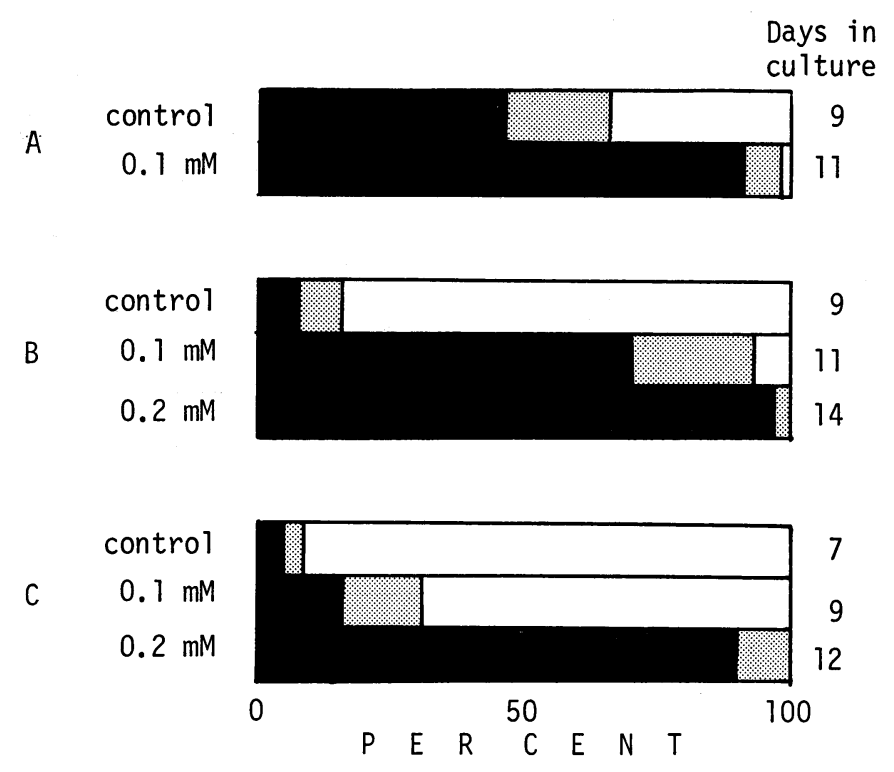

Fig. 2. Frequency distribution of microsprocytes with only univalent ( $)$, univalent and bivalent ( leptonema (B) or late leptonema to early zygonema (C) to NAL media at the concentrations indicated then cultured for the given periods prior to examination. As NAL has a marked effect on the meiotic rate (see Fig. 1), NAL-treated cells were sampled at the stages desired based on the concentrationdependent developmental periods measured in several experiments. Each value is the average for 2 to 8 buds in which 30 to 100 cells at diakinesis or metaphase I were measured.

produces achiasmatic division is present when cells reach late prophase (Fig. 3b). During the division process, chromosomes have a typical meiotic morphology, but they are present as univalents in the diploid number (24).

These univalents do not undergo normal anaphase segregation, but frequently their chromatids fall apart and are randomly distributed in the cell. Eventually an abnormal cytokinesis occurs resulting in cells with various numbers and sizes of nuclei. The failure of normal segregation probably is not due to defects in the spindle because in cells that have a mixture of bivalents and univalents, the bivalents undergo normal anaphase separation (Fig. 3c). Occasionally, the bivalents do not undergo anaphase separation nor do they desynapse; they fuse.

Chiasma frequency per chromosome in NAL-treated cells that have bivalents was not scored in this study, but qualitative observations of diakinesis nuclei gave the impression that cells are not restored to normal chiasma frequencies.

Pairing in meiocytes exposed to NAL. To determine whether the univalency induced by NAL is attributable to the failure of pairing or of chiasma formation, we exposed microsporocytes in early leptonema to concentrations of 0.1 to $0.4 \mathrm{mM} \mathrm{NAL}$ for appropriate periods then examined the cells for the synaptinemal complex (SC). Thin sections of untreated cells had numerous chromatin masses with the SC, whereas only small masses with the SC were found in sections of NAL-treated cells. There was no difference in the appearance and dimensions of the control and NAL-treated SCs. Typical nuclei in both types of cell at late zygotene are shown in Fig. 4.

The relative abundance of SC structures in both types with late zygotene nuclei is 


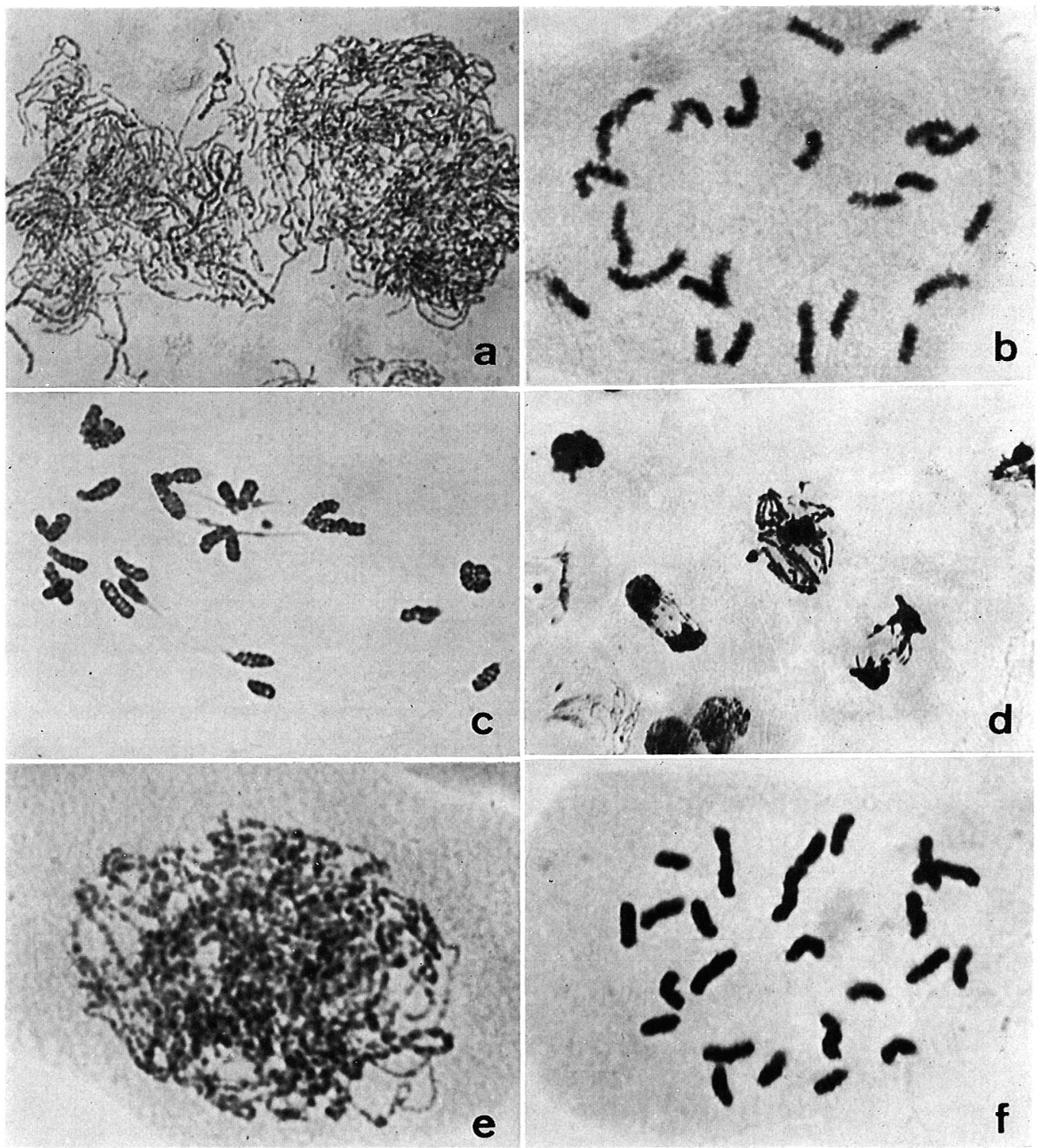

Fig. 3. Cytological appearances of lily microsporocytes cultured with NAL (a-d) or NOV (e, f). a : cells explanted at early leptonema and treated with $0.2 \mathrm{mM}$ NAL for 7 days. Very little pairing is present $\times 400$. b: $0.1 \mathrm{mM}$ NAL and an 11-day-treated cell showing 24 univalents at diakinesis, $\times 400$. c: $0.2 \mathrm{mM}$ NAL and a 12-day-treated cell showing bivalents and univalents at anaphase. Two bivalents remain unseparated, whereas most univalents are migrating to the poles, $\times 400$. $d$ : cells explanted at late leptonema and treated with $0.2 \mathrm{mM}$ NAL for 13 days showing chromosome stickiness. $\times 200$. e: a cell explanted at early leptonema and cultured for 9 days showing partial pairing, $\times 660$. f: a cell explanted at early leptonema and cultured for 11 days showing 24 univalents at diakinesis, $\times 400$.

shown in Fig. 5. A significant difference was found between the proportion of chromatin masses with SC segments and the total chromatin masses of both cell types. NAL-treated cells had markedly few SC-chromatin masses; about $10 \%$ at $0.1 \mathrm{mM}$ and 


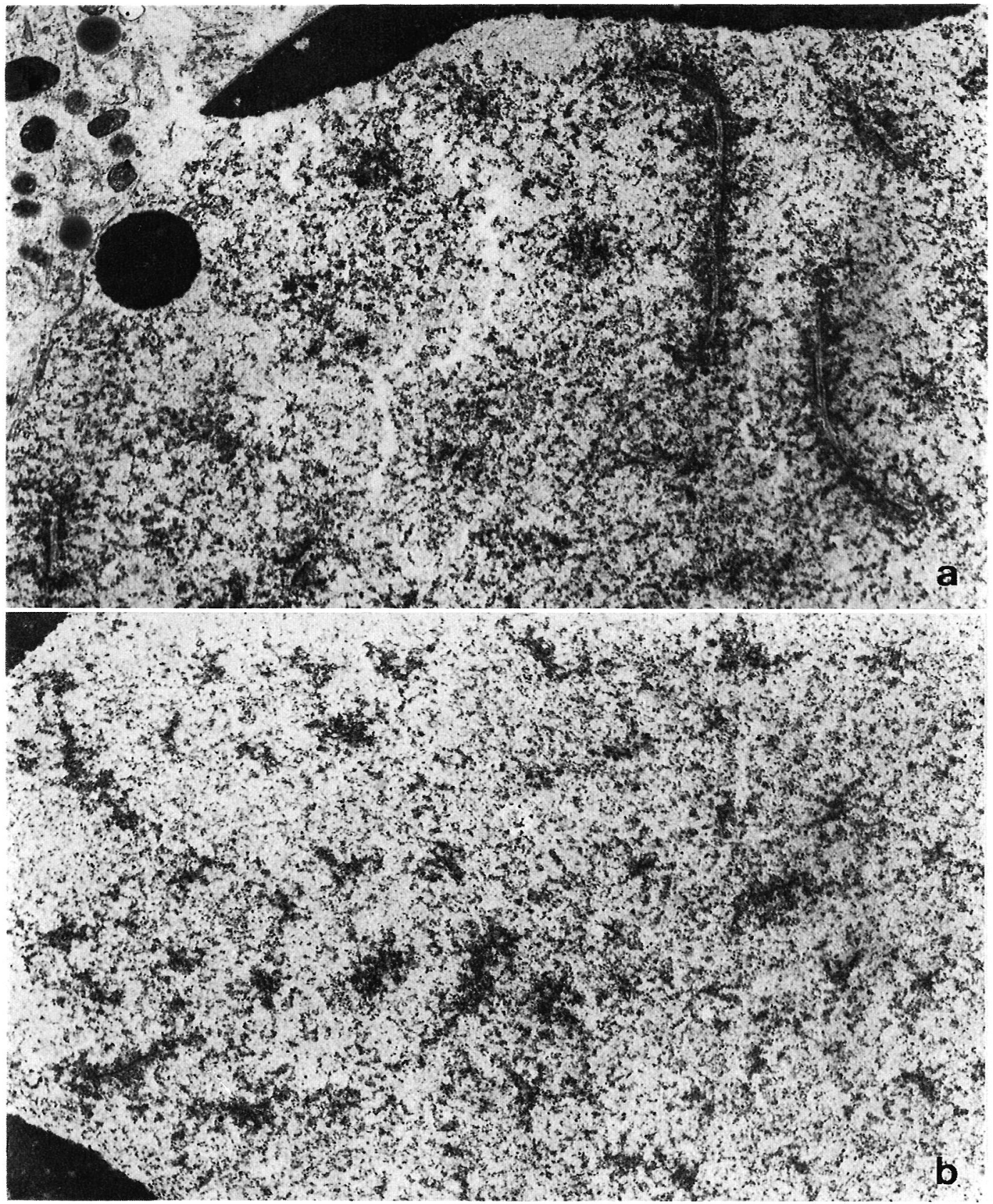

Fig. 4. Electron micrographs of cells explanted at early leptonema and cultured for 5 days in the absence (a) or presence (b) of $0.1 \mathrm{mM}$ NAL. a: zygotene nucleus showing numerous chromatin masses with a SC. b: nucleus showing a few fragments of the axial core but no SC structure. The degree of chromosome condensation is similar to that in early zygonema. $\times 11400$.

less than $5 \%$ at $0.2 \mathrm{mM}$. The mean value for SC content in control cells (about $35 \%$ ) must reflect full, or almost full, pairing. If so, this means that the $\mathrm{SC}$ content of 

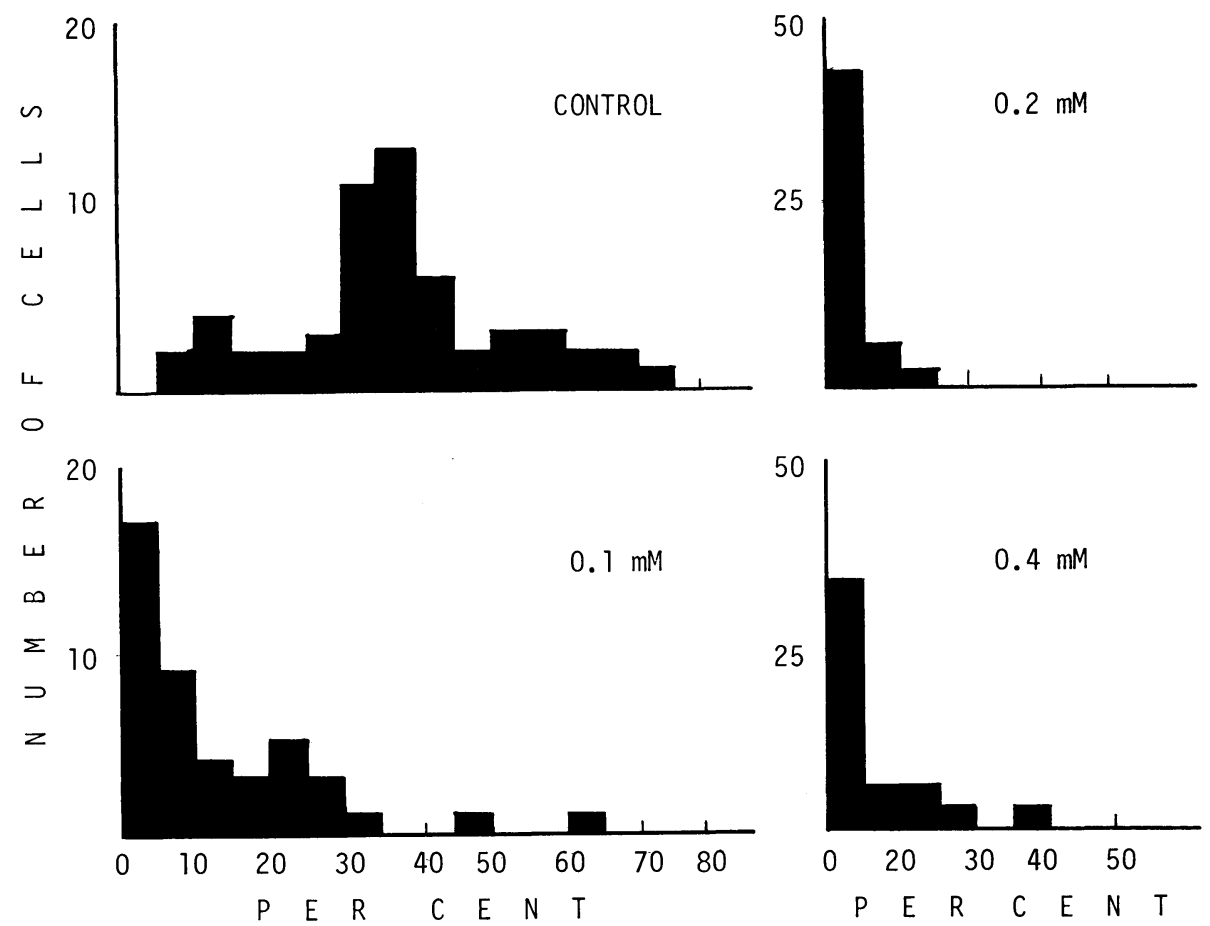

Fig. 5. The relative abundance of SCs in $0.1,0.2$ and $0.4 \mathrm{mM}$ NAL-treated and control microsporocytes at zygotene. Cells were explanted in late $\mathrm{G}_{2}$ or early leptonema and cultured for 5 days. The SC contents of 50 nuclei from each of the four groups were calculated. Values are the percent of chromatin masses with SC segments compared to the total chromatin masses present. The control and $0.1 \mathrm{mM}$ NAL-treated cells were obtained from one bud, the 0.2 and $0.4 \mathrm{mM}$ NAL-treated cells from other buds.

NAL-treated cells was less than $30 \%$ that of the controls.

Whether the abundance of SC at pachytene in these cells corresponds to the abundance of chiasmata at diakinesis is not clear. A comparison of the data shown in Fig. 2 with the data in Fig. 5, shows that the SC content is proportional to the bivalency, the ranges of which are attributable to the small degree of asynchrony in the cell population. The ineffectiveness of the pairing in low chiasma frequency, as has been observed in meiocytes of Black Beauty lilies (17), was not satisfactorily documented. Probably, paired chromosomes associated with the SC structure do assure bivalency in NAL-treated cells of Georgia lilies. If they do, the intensified achiasmate condition found on treatment with NAL would be due to the marked decrease in synapsis.

The interval necessary to induce univalency by NAL. Microsporocytes in early leptonema that were exposed to NAL showed enhanced susceptibility to chromosome pairing, but those in early zygonema or ongoing synapsis were unaffected. This means that the production of univalent chromosomes is related to the suppression of events essential for the initiation of synapsis; it is not an immediate consequence of the inhibition of DNA synthesis.

We have no evidence of the primary lesions that would lead to a correspondingly asynaptic types of chromosome, but the nature of such lesions would be made clear if the interval needed to induce univalency could be determined, or if the possibility 
of repairing the primary lesions induced by NAL could be studied.

Our experiments were done with microsporocytes that first had been exposed to $0.2 \mathrm{mM}$ NAL for different periods then transferred to basic medium. Cells that had been explanted in either early leptonema or mid to late leptonema were observed. The value $0.2 \mathrm{mM}$, is the effective concentration for the induction of univalency in leptonema cells exposed continuously (Table 1). Fig. 6 shows the percentage of cells with only univalent chromosomes to the total cells in early leptonema exposed to $0.2 \mathrm{mM}$ NAL for 2 to 6 days before transfer to normal medium. If exposed for 2 days cells resumed normal development. Pulse treatment for 6 days totally inhibited the production of bivalents. An appreciable increase in the number of cells with univalent chromosomes was found when transfer was made after 3 days of exposure. In the 4-day treatment, the frequency of cells with only univalents was about $40 \%$, a mixture of univalents and bivalents being frequently produced (about $35 \%$ ).

The cytological progress of the cells in NAL media showed that the cells were still in the early zygonema stage at that time. Thus, the conclusion is that the high incidence of univalency in cells that were removed from NAL after 4 days is the result of the specific action of NAL at the initiation of synapsis. The exact stage has yet to be determined, but the stage at which transient exposure to NAL causes a disturbance of chromosome pairing is somewhere in the period spanning late leptonema to early zygonema. In all cases, the production of univalents by NAL was restricted to cells treated at late leptonema or earlier. Cells treated with NAL at stages later than early zygonema produced few univalents. Apparently, NAL can prevent synapsis if cells are exposed to the chemical prior to the initiation of pairing, but it cannot do so if cells are exposed after pairing is relatively complete.

Effects of NOV on meiotic cells. To obtain more information on the action of NAL on synaptic events, we examined lily microsporocytes exposed to NOV, a chemical known to have effects similar to NAL in prokaryote systems. Microsporocytes in early leptonema were treated with concentrations of 0.005 to $0.02 \mathrm{mM}$ NOV for various periods. At $0.005 \mathrm{mM}$, meiotic development was slightly reduced but showed

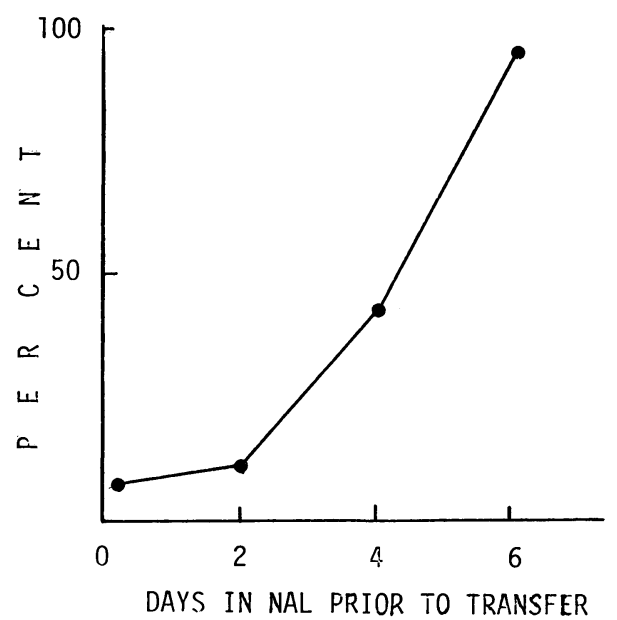

Fig. 6. Reversibility of the effects of NAL on the induction of univalency in lily microsporocytes. Cells were explanted to an NAL $(0.2 \mathrm{mM})$ medium at early leptonema then after one or more days of culture were transferred to basic medium. Frequencies are given as the percent of cells with only univalent chromosomes in comparison to the total number of cells. 
no gross cytological abnormalities; cells reached metaphase $I 1$ to 2 days later than the controls. Chromosome pairing and chiasma formation were similar to those in the control cells. At $0.01 \mathrm{mM}$, cells reached metaphase I after 10 to 12 days of culture, 3 to 5 days later than the controls. Most cells underwent partial pairing at zygonema (Fig. 3e) and had high frequencies of univalent chromosomes at diakinesis (Fig. 3f). The frequencies of cells either with univalents or bivalents, or both was similar to those of cells treated with $0.1 \mathrm{mM}$ NAL.

A concentration of $0.02 \mathrm{mM}$ NOV caused extreme inhibition of meiotic development, suppressed cells remaining suspended in some stage of the meiotic prophase after 2 weeks of culture. The few cells that reached late prophase during this period showed relatively high chromosome stickiness.

These results show that partial inhibition of DNA synthesis by NOV does not primarily affect chromosome structure but does drastically affect the synaptic event. The correspondence of abnormal patterns in meiotic cells exposed to NAL and cells treated with NOV suggests that these drugs act on a common feature of chromosome organization or that primary lesions are common to both.

\section{DISCUSSION}

The small amount of DNA synthesized during zygonema represents delayed replication of part of the chromosomal DNA $(5,10,15,16)$, and this zDNA synthesis is considered to function in the synaptic process $(7,12,13)$. On the basis of biochemical and cytological studies of various inhibitors of DNA synthesis, assumptions have been made that initiation of DNA synthesis during zygonema is necessary for the pairing of chromosomes and that the completion of DNA synthesis is required for the maintenance of chromosome integrity.

NAL and NOV are known selective inhibitors of DNA synthesis that suppress the activity of DNA gyrase in prokaryotes (1). Although this enzyme activity has not been demonstrated in higher plant cells, the effects of these drugs on lily microsporocytes clearly were circumscribed in this study. Chromosome condensation, spindle function, and cell wall formation were scarcely affected, but the meiotic rate and some features of chromosome organization were profoundly affected. These effects are assumed to result from the partial inhibition of DNA synthesis. Circumstantial evidence that supports this assumption is considerable. The types of abnormalities induced by these drugs correspond to abnormalities induced by other DNA-inhibitory agents; the delay in meiotic development is induced only during DNA synthesis; and also inhibition of prophase DNA synthesis by NAL has been demonstrated chemically in our laboratory. The chemical data will be reported in detail elsewhere, but briefly the incorporation of radioactive thymidine into DNA, when $0.2 \mathrm{mM} \mathrm{NAL}$ is added to leptonema cells for 2 days produces about $50 \%$ inhibition of DNA synthesis, and $0.4 \mathrm{mM}$ produces about $70 \%$ inhibition. We have no answer as to how NAL interferes on the molecular level with DNA replication; there are various possibilities for the action of NAL. Regardless of the nature of its effectiveness, the effects found in this study can be regarded as adequate proof that NAL actually inhibits zygotene DNA synthesis, and that this eventually produces cytological abnormalities in meiotic cells.

In comparison to the response of meiotic cells to other DNA-inhibitory agents, NAL and NOV on leptonema cells are notable for their induction of univalency without visible chromosome aberrations. We have applied various inhibitors to meiotic 
cells in different stages of meiosis and followed the cytological consequences $(7,13,18)$. The effectiveness of the inhibitors on meiosis could be divided into two general categories, the AdR- and HU-types.

Most agents are of the AdR-type and usually induce irreversible blockage in meiotic development or severe chromosome aberrations, the initiation of SC formation and ongoing synapsis being affected. HU-types have no general toxic effect on chromosomes. Even when these inhibitors are applied, cells can pass through prophase, and synapsis develops normally. When treated continuously with NAL, meiotic cells showed no enhanced susceptibility to chromosome fragmentation or breakage, but a major effect was the disturbance of chromosome pairing. This means that like HU, NAL does not directly affect chromosome integrity; it suppresses the rate of zDNA synthesis. Even though chromosome integrity is exclusively DNA-dependent, simple prolongation of the period of synthesis may not lead to incomplete DNA synthesis. Chromosome abnormalities attributable to gaps along the DNA chain that would ultimately lead to breaks are not found in NAL-treated cells. Therefore, cells treated with NAL and NOV would not leave the regions of delayed synthesis until zygonema in the chromosome incompletely replicated.

When several inhibitors of DNA synthesis were used at the half concentrations that induced marked suppression of meiotic development, as described previously (13), cells usually responded only with a delay in meiotic progression. In spite of partial inhibition of zDNA replication, they showed typical chromosome pairing. This suggests that initiation of DNA synthesis during zygonema is required for the initiation of chromosome pairing. On this assumption, the principal question produced by this study is what is the specific nature of the actions of NAL and NOV in the induction of asynapsis. A variety of possibilities are conceivable for the underlying mechanism that produces abnormalities. An essential feature may be the coordination of DNA replication with chromosome alignment; this might facilitate the further process of synapsis.

In the synaptic process, alignment of homologous sites of chromosomes is considered as a transient event which leads to synapsis. If the initiation of DNA synthesis is not coordinated with this alignment, an asynaptic condition would result. Two possibilities that might render otherwise normal meiotic cells asynaptic are premature or delayed replications of zDNA against the timing of alignment during zygonema.

Premature replication in the presynaptic phase has been found in meiotic cells explanted during the $\mathrm{G}_{2}$ phase of premeiosis; these cells are asynaptic or highly achiasmatic when they reach diplonema. A delay in replication also is likely because of the inhibitory properties of these drugs, but our attempts to demonstrate a delay in the initiation of DNA synthesis with biochemical techniques so far have given equivocal results.

An alternative interpretation of the effects of NAL and NOV is that disturbance of organized chromosome condensation during the presynaptic process makes alignment of homologous sites within the chromosomes impossible. Because chromosomes are partly condensed before pairing begins, condensation must be highly organized to expose sites that could serve to align homologs. Since zDNA synthesis has been reported to occur close to or within the SC (10), it is likely that zDNA sequences occupy the presumed matching sites. If so, the effectiveness of these drugs on late leptonema cells suggests that the drugs interfere with chromosome condensation during the presynaptic process. 
Although the reported experiments did not determine the exact role that zDNA replication plays in the synaptic process, they did show that a discrete interval follows the partial condensation of chromosomes during which time synapsis may be effectively inhibited by suppressing DNA synthesis. A more detailed cytological examination combined with biochemical studies should provide strong circumstantial evidence for the significance of zDNA replication to the synaptic process.

Acknowledgements. I thank Dr. Michio Ito for his expert advice and encouragement during this study and for his critical reading of this manuscript.

\section{REFERENCES}

1. Champoux, J.J. Proteins that affect DNA conformation. Ann. Rev. Biochem. 47, 449-479, 1978

2. ERICKSON, R.O. Cytological and growth correlations in the flower bud and anther of Lilium longiflorum. Amer. J. Bot. 35, 729-739, 1948

3. Holm, P.B. The premeiotic DNA replication of euchromatin and heterochromatin in Lilium longiflorum (Thumb.). Calsberg Res. Commu. 42, 249-281, 1977

4. Hotta, Y., M. Ito and H. Stern. Synthesis of DNA during meiosis. Proc. Natl. Acad. Sci. U.S.A. 56, 1184-1191, 1966

5. Hotta, Y. and H. Stern. Analysis of DNA synthesis during meiotic prophase in Lilium J. Mol. Biol. 55, 337-355, 1971

6. Iто, M. and Y. HоттA. Radioautography of incorporated ${ }^{3} \mathrm{H}$-thymidine and its metabolism during meiotic prophase in microsporocytes of Lilium. Chromosoma (Berl.) 43, 391-398, 1973

7. Ito, M., Y. Hотta and H. STERn. Studies on meiosis in vitro II. Effect of inhibiting DNA synthesis during meiotic prophase on chromosome structure and behavior. Develop. Biol. 16, 54-77, 1967

8. Ito, M. and H. Stern. Studies of meiosis in vitro I. In vitro culture of meiotic cells. Develop. Biol. 16, 36-53, 1967

9. Ito, M. and M.H. Takegami. Commitment of mitotic cells to meiosis during the $\mathrm{G}_{2}$ phase of premeiosis. Plant \& Cell Physiol. 23, 943-952, 1982

10. Kurata, N. and M. Ito. Electron microscope autoradiography of ${ }^{3} \mathrm{H}$-thymidine incorporation during the zygotene stage in microsporocytes of lily. Cell Struc. Funct. 3, 349-356, 1978

11. Parchman, L.G. and H. Stern. The inhibition of protein synthesis in meiotic cells and its effect on chromosome behavior. Chromosoma (Berl.) 26, 298-311, 1969

12. Rотн, T.F. and M. Iто. DNA-dependent formation of synaptinemal complex at meiotic prophase. J. Cell Biol. 35, 247-255, 1967

13. Sakaguchi, K., N. Kurata, M.H. Takegami and M. Ito. Inhibition of DNA and RNA syntheses and suppression of meiotic development in lily microsporocytes. Cell Struc. Funct. 5, 367-377, 1980

14. SEN, S.K. Chromatin-organization during and after synapsis in cultured microsporocytes of Lilium in presence of mitomycin C and cycloheximide. Exp. Cell Res. 55, 123-127, 1969

15. Stern, H. and Y. Hotta. Biochemical controls of meiosis. Ann. Rev. Genet. 7, 37-66, 1973.

16. Stern, H. and Y. HotTA. The organization of DNA metabolism during the recombinational phase of meiosis with special reference to humans. Mol. Cellul. Biochem. 29, 145-158, 1980

17. Toledo, L.A., M.D. BennetT and H. Stern. Cytological investigations of the effect of colchicine of meiosis in Lilium hybrid cv. "Black Beauty" microsporocytes. Chromosoma (Berl.) 72, 157173, 1979

18. Takegami, M.H. and M. Ito. Effect of hydroxyurea on mitotic and meiotic divisions in explanted lily microsporocytes. Cell Struct. Funct. 7, 29-38, 1982

19. TaKegami, M.H., M. Yoshioka, I. Tanaka and M. Ito. Characteristics of isolated microsporocytes from liliaceous plants for studies of the meiotic cell cycle in vitro. Plant \& Cell Physiol. 22, 1-10, 1981 